\title{
A Quality by Design (QbD) Framework for Reversed-Phase Liquid Chromatography Method Development
}

\author{
Yuan Huang* \\ Research Scientist, Human Biology \& Data Science Engine, Eisai Inc, 4 Corporate Dr, Andover 01810, MA, USA
}

Received: May 07, 2016; Accepted: June 28, 2016; Published: July 03, 2016

*Corresponding authors: Research Scientist, Human Biology \& Data Science Engine, Eisai Inc, 4 Corporate Dr, Andover 01810, MA, USA, E-mail: Yuan_Huang@Eisai.com

\begin{abstract}
A framework for reversed-phase HPLC method development was developed by combining computer simulation, UPLC scouting experiments and surface response design. An interactive computer simulation program was developed for optimizing the gradient elution conditions. This simulation program integrated the kinetics and flow resistance parameters of 18 commonly used columns. Accurate prediction of retention times by this simulation program was demonstrated in this work. This program was used to propose the optimum gradient operational conditions under given constraints, which greatly reduced the time required for method development. The Box-Behnken design was used to evaluate the optimum operational conditions obtained from the simulation and experiments and define the method operable design space. Further insights into the separation mechanisms were obtained from the statistical analysis results of DOE.
\end{abstract}

Keywords: HPLC method development; Computer simulation; DOE; surface response design; Box-Behnken design; statistical analysis

\section{Introduction}

The concept of Quality by Design (QbD) has been well defined and described for the development and manufacture of pharmaceutical drug substances and drug product [1-3]. According to ICH Q8 [1], design space and manufacturing controls are established based on the knowledge gained from pharmaceutical development. Once the proposed design space is approved by regulatory, operational and manufacturing parameter changes within this design space are not considered as a change, and consequently, would not initiate regulatory post approval change processes.

The same QbD principles have been applied to the HPLC method development [4-6]. Using Design of Experiment (DOE) technique, the Method Operable Design Region (MODR) can be established. Operating the method within this MODR will guarantee that the Critical Quality Attributes (CQAs) of the method, such as separation resolution and peak tailing factors, can be controlled within a reasonable range, resulting in a robust method that consistently delivers the intended performance. In addition, by DOE based strategies, detailed information about how the operational parameters affect the CQAs within a narrow region near the optimum operational conditions can be easily obtained using statistical analysis methods [7], which not only provides information for the quantitative evaluation of the method robustness, but also provides critical information for the fundamental study of the separation mechanisms.

Although numerous papers have been published on reversed phase HPLC method development and optimization by DOE strategies [5,8-10], method development strategies that fully rely on DOE need to search the entire high dimensional design space, which usually requires large amount of experimental trials, especially for complex samples.

Computer simulation has been widely used in HPLC method development and has been proved to be highly efficient to find the optimum operational conditions [11-25]. By allowing DOE to only study the narrow region of the design space near the optimum, computer simulation can greatly speed up the process for defining the MODR by DOE [20]. It should be noted that HPLC method development is a complicated process where many operational parameters, including column selectivity parameters, column kinetic properties, mobile phase compositions, column temperature, ion pair types and concentration, gradient profile, and their interactions need to be considered. Although computerassisted method development methods can greatly speed up the method development process, understanding of the fundamental separation mechanisms and the operators' method development experience are still critical to method development.

In this paper, a framework is reported that combines the computer simulation and surface response design for HPLC method development. The goals of this manuscript are the following: First, to demonstrate that by combining computer simulation and surface response design, HPLC methods can be effectively developed for challenging separation problems by using reasonable amount of experimental trials; Second, to demonstrate that UHPLC/UPLC can be used as an efficient tool for conventional HPLC method development. Although UHPLC/UPLC has been widely used in pharmaceutical industry, conventional HPLC is still used in many cases due to instrument compatibility and method transfer issues. This work showed 
that the solute retention parameters obtained from an acquity BEH C18 UPLC column operated on a UPLC system can be directly applied to X Bridge C18 column for conventional HPLC method development by computer simulation. Due to the faster separation speed $[26,27]$. And lower mobile phase consumption of UHPLC/UPLC compared to conventional HPLC, this can greatly reduce the time and cost required for conventional HPLC method development; Third, to demonstrate that surface response design together with the following statistical analysis not only provides a critical tool to quantitatively evaluate the robustness of HPLC methods and define the MODR, but also provides further insights into the fundamental mechanisms of the separation. Finally, to demonstrate an interactive simulation program that integrated various column parameters for gradient method development. The Graphic User Interfaces (GUI) of this tool makes it easy to use for users without in-depth knowledge and understanding of gradient elution theory and simulation. Results of this work showed this simulation program can accurately predict the retention times and propose the optimum operational conditions based on the constraints set up by users.

It should be noted that this manuscript only discusses the framework for finding the operational conditions that satisfy the resolutions and peak shape quality attributes, and method validation is beyond the scope of this work.

\section{Materials and Methods}

\section{Chemicals}

All solvents used in this work were HPLC grade. Acetonitrile (ACN) and Methanol (MeOH) were purchased from EMD. Formic acid (FA) was purchased from Fluka. Trifluoro acetic acid (TFA) was obtained from Thermo Scientific. Ammonium hydroxide ( $\mathrm{NH}_{4} \mathrm{OH}, \mathrm{ACS}$ reagent grade) was purchased from Sigma-Aldrich. HPLC water was obtained from a Millipore system. Three aqueous mobile phases $(0.1 \%$ TFA; $0.1 \%$ formic acid; and $0.1 \%$ ammonium hydroxide), and two organic modifiers (acetonitrile and methanol) were utilized in the method development.

Solutes used in the isocratic flow study were obtained from Sigma-Aldrich. The sample mixture was prepared by appropriately diluting individual stock solutions of the solutes in 40:60 acetonitrile-water, resulting in a sample mixture solution containing acetophenone $(0.2 \mathrm{mg} / \mathrm{mL})$, propiophenone $(0.4$ $\mathrm{mg} / \mathrm{mL})$, butyrophenone $(1.0 \mathrm{mg} / \mathrm{mL})$ and valerophenone $(1.0 \mathrm{mg} / \mathrm{mL})$. Uracil was purchased from Sigma and dissolved in pure water at a concentration that gave an adequate signal to determine the column dead volume.

A mixture sample consisting of Active Pharmaceutical Ingredient (API) and process impurities developed by Eisai Inc. was used for the method development. The molecular weights of API and major impurities were measured by LC-MS.

\section{Instruments and columns}

The UPLC/UHPLC experiments were conducted using a Waters Acquity UPLC I class system equipped with a single column heating compartment, a photodiode-array detector and a SQD MS detector. Detection wavelength was set at $240 \mathrm{~nm}$ for alkylphenones and $260 \mathrm{~nm}$ for the API mixture sample. The SQD was set to scan mode from $150-800 \mathrm{Da}$. The capillary voltage was set at $3.0 \mathrm{KV}$, cone voltage $30 \mathrm{~V}$, extractor voltage $3 \mathrm{~V}$, source temperature was $150{ }^{\circ} \mathrm{C}$, and the desolvation temperature was $350^{\circ} \mathrm{C}$.

The HPLC experiments were conducted using an Agilent 1100 HPLC system equipped with a column heating compartment and a VWD UV detector. Detection wavelength was set at $260 \mathrm{~nm}$ for the pharmaceutical mixture sample and $240 \mathrm{~nm}$ for alkylphenones.

The instrument gradient void volumes were measured to be $114 \mu \mathrm{L}$ and $1.33 \mathrm{~mL}$ for UPLC and HPLC systems, respectively.

Eighteen columns commonly used by the Eisai Inc. Analytical Chemistry team for method development were characterized by flow study. The names, dimensions, pore diameters, and particle sizes of these columns are listed in table 1.

\section{Software and Data Treatment}

For all the experiments conducted on both UPLC and HPLC systems, instrument control and data analysis were performed by Waters Empower II software (Milford, MA USA). Column van Deemter and flow resistance parameters were obtained by fitting the flow study data using Microsoft Excel (Bellevue, WA). A Microsoft Excel simulation program coded by Visual Basic for Application (VBA) was used for modeling and optimizing the gradient elution conditions. Column van Deemter and flow resistance parameters were stored in a MySQL (Redwood, CA USA) database. The VBA simulation program communicated with the MySQL database by ODBC, and the column parameters required for modeling were acquired by the VBA program from MySQL using standard SQL queries. JMP software V 9.0 was used for the DOE work.

\section{Flow study experiments}

Flow studies were performed on columns listed in Table 1 with alkylphenones. For UPLC/UHPLC columns, plate counts based on butyrophenone were measured at a series of flow rates between 0.05 and $1.0 \mathrm{~mL} / \mathrm{min}$, while for HPLC columns, flow rates were varied between 0.1 and $2.0 \mathrm{~mL} / \mathrm{min}$. All the flow study experiments were carried out using 50/50 water/acetonitrile isocratic elution, and the columns were thermo stated at $35^{\circ} \mathrm{C}$. In addition, all the measured plate counts were corrected for extracolumn volume and broadening effects, which were measured by injecting butyrophenone with a zero-dead-volume connector in place of the column. The plate heights for the flow curve were calculated using the corrected plate counts.

In addition to the plate counts, column pressure for each column at each flow rate was recorded. These column pressures, after corrected for instrumental pressure at the corresponding flow rates, were used to calculate the column flow resistance.

\section{Mobile phase and column screening}

Zorbax Bonus RP RRHD (pore size: $80 \AA$, particle diameter: $1.8 \mu \mathrm{m}$ ), Zorbax Extend C18 RRHD (pore size: $80 \AA ̊$, particle 
Table 1: Summary of the columns characterized by the flow study.

\begin{tabular}{|l|l|l|l|l|}
\hline Column Name & Column I.D. $\mathbf{( m m})$ & Column Length $(\mathbf{c m})$ & Pore diameter $(\AA)$ & Particle Diameter $(\boldsymbol{\mu m})$ \\
\hline Zorbax Bonus RP RRHD & 2.1 & 5 & 80 & 1.8 \\
\hline Zorbax Extend C18 RRHD & 2.1 & 5 & 80 & 1.8 \\
\hline ZorbaxStableBond C18 RRHD & 2.1 & 5 & 80 & 1.8 \\
\hline Acquity BEH C18 & 2.1 & 5 & 130 & 1.7 \\
\hline Acquity CSH C18 & 2.1 & 5 & 130 & 1.7 \\
\hline Acquity BEH Shield RP18 & 2.1 & 5 & 90 & 1.7 \\
\hline Acquity HSS T3 & 2.1 & 5 & 130 & 3.8 \\
\hline XBridge C18 & 4.6 & 15 & 130 & 3.5 \\
\hline XBridgeBEH Shield RP18 & 4.6 & 15 & 3 \\
\hline Atlantis T3 & 4.6 & 10 & 3.5 \\
\hline Sunfire C18 & 4.6 & 15 & 100 & 5 \\
\hline Symmetry Shield RP18 & 4.6 & 15 & 100 & 3 \\
\hline Luna C18(2) & 4.6 & 10 & 80 & 3.5 \\
\hline Zorbax Eclipse XDB-C18 & 4.6 & 15 & 80 & 3.5 \\
\hline Zorbax Eclipse XDB-Phenyl & 4.6 & 15 & 80 & 3.5 \\
\hline Zorbax Bonus RP & 4.6 & 15 & 80 & 3.5 \\
\hline Zorbax Stable Bond 80A C18 & 4.6 & 15 & 80 & 3 \\
\hline YMC-Pack Pro C18 RS & 4.6 & 15 & \\
\hline
\end{tabular}

diameter: $1.8 \mu \mathrm{m}$ ), Zorbax Stable Bond C18 RRHD (pore size: 80 Å, particle diameter: $1.8 \mu \mathrm{m}$ ), Acquity BEH C18 (pore size: $130 \AA$ particle diameter: $1.7 \mu \mathrm{m}$ ) and Acquity BEH Shield RP18 (pore size: $130 \AA$, particle diameter: $1.7 \mu \mathrm{m}$ ) columns were used for the column screening. All the columns had the same dimension of 2.1 $\times 100 \mathrm{~mm}$.

Due to the severe peak tailing observed when $0.1 \%$ FA aqueous solution was used in the mobile phase, only mobile phases consisting of methanol- $0.1 \%$ TFA aqueous, methanol-0.1\% $\mathrm{NH}_{4} \mathrm{OH}$ aqueous $(\mathrm{pH}=10.5), \mathrm{ACN}-0.1 \%$ TFA aqueous and $\mathrm{ACN}-0.1 \% \mathrm{NH}_{4} \mathrm{OH}$ aqueous ( $\left.\mathrm{pH}=10.5\right)$ were fully screened. The methanol- $0.1 \% \mathrm{NH}_{4} \mathrm{OH}$ aqueous and $\mathrm{ACN}-0.1 \%$ $\mathrm{NH}_{4} \mathrm{OH}$ aqueous mobile phases were only used on Zorbax Extend C18 RRHD and Acquity BEH C18 columns since only these two columns are compatible with basic mobile phase conditions. The ACN-0.1\% TFA aqueous and methanol- $0.1 \%$ TFA aqueous mobile phases were screened for all the five columns.

For each column under a given mobile phase composition condition, three gradient scouting runs with different gradient times were completed. The gradient started at 75:25\% aqueous: organic and ramped to $10: 90 \%$ aqueous: organic for all three runs. The flow rates were fixed at $0.5 \mathrm{~mL} / \mathrm{min}$. The gradient times of these runs were 6, 18 and 30 minutes, respectively. The API and seven other impurity peaks that distributed within the entire gradient time window were chosen to represent the API mixture sample for the gradient condition optimization (see Figure 1). For a given column-mobile phase combination, if reasonable resolutions of the critical pairs were observed during the scouting runs, retention times of API and the seven impurities obtained from the three scouting runs were submitted to the Excel simulation software for optimization. Based on the retention times of API and the seven impurities under the three gradient scouting run conditions, the retention parameters $\left(\mathrm{k}_{\mathrm{w}}^{\prime}\right.$

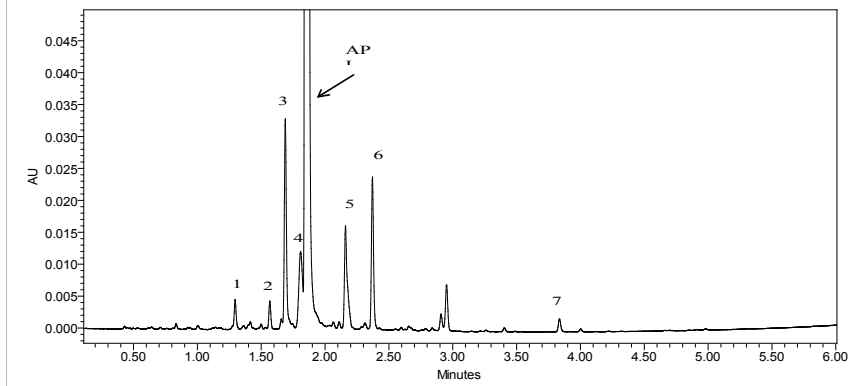

Figure 1: Chromatogram of API mixture sample containing API and seven impurities chosen for method development by

Simulation program.

Gradient conditions: B\% increased from 25\% to $90 \%$ linearly in 6 minutes. Solvent A: $0.1 \%$ TFA in water. Solvent B: acetonitrile. Flow rate = $0.5 \mathrm{~mL} / \mathrm{min}$. Detection wavelength was set at $260 \mathrm{~nm}$. Injection volume was $0.5 \mu \mathrm{L}$. The column temperature was fixed at $35^{\circ} \mathrm{C}$. The separation was carried out on a 2.1 I.D. $\times 100 \mathrm{~mm}$ BEH-C18 column packed with $1.7 \mu \mathrm{m}$ particles.

and S values) of each peak were calculated and used by the simulation software to predict the retention times and peak widths, and optimize the gradient conditions based on the linear solvent strength theory $[24,25,28,29]$.

\section{Procedure for DOE data analysis by JMP}

Once operational conditions with acceptable resolutions were obtained from simulation, the conditions were confirmed by experiments. In addition, experiments were performed to further optimize the conditions to make sure that the maximum column pressure, signal intensity and resolutions were within the appropriate ranges. After all these steps, the proposed operational conditions were defined. Box-Behnken design was then used to evaluate how the changes in this proposed operational conditions affected the CQAs. The MODR was then 
defined based on the analysis results of the Box-Behnken design The Box-Behnken design table generated by JMP software based on the proposed operational conditions, and the corresponding experimental conditions of the 15 HPLC experimental trails were shown in table 6. Attributes including the retention times of API, impurity 4 and unknown impurity (defined in figure 5), values of API tailing factor and API peak width at half height, the resolution between API and impurity 4 (defined as resolution 1) and the resolution between API and unknown impurity (defined as resolution 2) for each HPLC experimental trial were measured from these15 experimental runs. Based on the experimental results, effects of the TFA\%, column temperature and flow rate on these attributes were analyzed, and regression models were established by JMP software. To establish the models, main effects and interactions were first analyzed and only those with p-values less than 0.05 were used to fit the regression models. All the models had R-square values higher than 95\%. The estimated value, standard error, and t-test results for model parameters were listed in table 6 . The general procedure of using JMP software to analyze the data and fit regression models were documented in chapters 5 and 6 of JMP software document [30].

\section{Results and Discussion}

\section{Flow study}

Flow study is a commonly used method to measure the kinetic properties of columns. The detailed discussion of the theory and procedure of flow study can be found in references [31-35]. In this work, plate height of each column obtained from flow study experiments based on butyrophenone and the corresponding reduced linear velocity were used to fit the van Deemter equation to obtain the van Deemter parameters. The mobile phase viscosity values for aqueous-methanol and aqueous-acetonitrile were calculate by equations reported previously [36] and solute diffusion coefficients were estimated by the method reported by Li and Carr $[37,38]$. The calculated van Deemter parameters are listed in table 2 . The interstitial porosity values for some of the columns were obtained from reported values [39-41] (see Table 3). A default value of 0.38 was used for columns that had no reported interstitial porosity values. In addition, based on the backpressure of each column measured at each flow rate, values of flow resistance were calculated using the nominal particle size and the interstitial porosity by the procedure reported by Zhan [34]. The column physical properties and the calculated flow resistance parameters are listed in table 3 . These parameters were stored in My SQL database, and used by the Excel simulation software for gradient operational condition optimization. It should be noted that the purpose of obtaining these column parameters was to obtain an accurate estimation of the separation efficiency and column pressure for an average column of its type by simulation, not for the fundamental understanding of the performance of these columns.

The author believes that integrating these column parameters in the simulation program will be helpful, especially for optimizing the separation conditions for complex samples where high peak capacities obtained by coupled column systems are needed $[34,39,42]$. In addition, integrating these column parameters in the simulation programs allows users to easily optimize both the gradient operational conditions and column types for HPLC and UPLC method development by using the user-friendly graphic user interface of the simulation program.

Although several papers have been published on optimizing HPLC/UPLC methods for complex samples based on carefully characterized columns using the flow study method reported in this manuscript $[34,42,43]$, these papers only characterized a few specific types of columns. As far as the author knows, this

Table 2: Summary of the van Deemter parameters of the columns in flow study.

\begin{tabular}{|c|c|c|c|c|}
\hline Column Name & A & B & C & S.E. ${ }^{a}$ \\
\hline Zorbax Bonus RP RRHD & $1.10 \pm 0.07$ & $10.78 \pm 0.34$ & $0.057 \pm 0.003$ & 0.02 \\
\hline Zorbax Extend C18 RRHD & $1.11 \pm 0.02$ & $9.97 \pm 0.08$ & $0.045 \pm 0.001$ & 0.007 \\
\hline ZorbaxStableBond C18 RRHD & $1.04 \pm 0.02$ & $11.41 \pm 0.11$ & $0.038 \pm 0.001$ & 0.009 \\
\hline Acquity BEH C18 & $0.38 \pm 0.06$ & $11.88 \pm 0.20$ & $0.069 \pm 0.003$ & 0.04 \\
\hline Acquity CSH C18 & $0.37 \pm 0.05$ & $12.51 \pm 0.17$ & $0.075 \pm 0.003$ & 0.04 \\
\hline Acquity BEH Shield RP18 & $0.37 \pm 0.05$ & $10.59 \pm 0.17$ & $0.081 \pm 0.003$ & 0.04 \\
\hline XBridge C18 & $0.75 \pm 0.03$ & $11.32 \pm 0.09$ & $0.048 \pm 0.002$ & 0.02 \\
\hline XBridgeBEH Shield RP18 & $0.93 \pm 0.07$ & $8.61 \pm 0.19$ & $0.033 \pm 0.003$ & 0.05 \\
\hline Atlantis T3 & $0.91 \pm 0.14$ & $16.48 \pm 0.34$ & $0.049 \pm 0.008$ & 0.12 \\
\hline Sunfire C18 & $0.64 \pm 0.07$ & $14.05 \pm 0.19$ & $0.052 \pm 0.004$ & 0.05 \\
\hline Symmetry Shield RP18 & $1.32 \pm 0.03$ & $7.36 \pm 0.10$ & $0.042 \pm 0.001$ & 0.02 \\
\hline Luna C18(2) & $1.06 \pm 0.09$ & $10.96 \pm 0.22$ & $0.030 \pm 0.005$ & 0.08 \\
\hline Zorbax Eclipse XDB-Phenyl & $0.72 \pm 0.04$ & $7.74 \pm 0.10$ & $0.052 \pm 0.002$ & 0.04 \\
\hline Zorbax Bonus RP & $1.01 \pm 0.03$ & $6.58 \pm 0.08$ & $0.054 \pm 0.002$ & 0.03 \\
\hline ZorbaxStableBond 80A C18 & $0.70 \pm 0.04$ & $9.48 \pm 0.09$ & $0.051 \pm 0.002$ & 0.03 \\
\hline YMC-Pack Pro C18 RS & $1.26 \pm 0.17$ & $9.72 \pm 0.67$ & $0.108 \pm 0.009$ & 0.05 \\
\hline
\end{tabular}


Table 3: Physical properties of the columns in flow study.

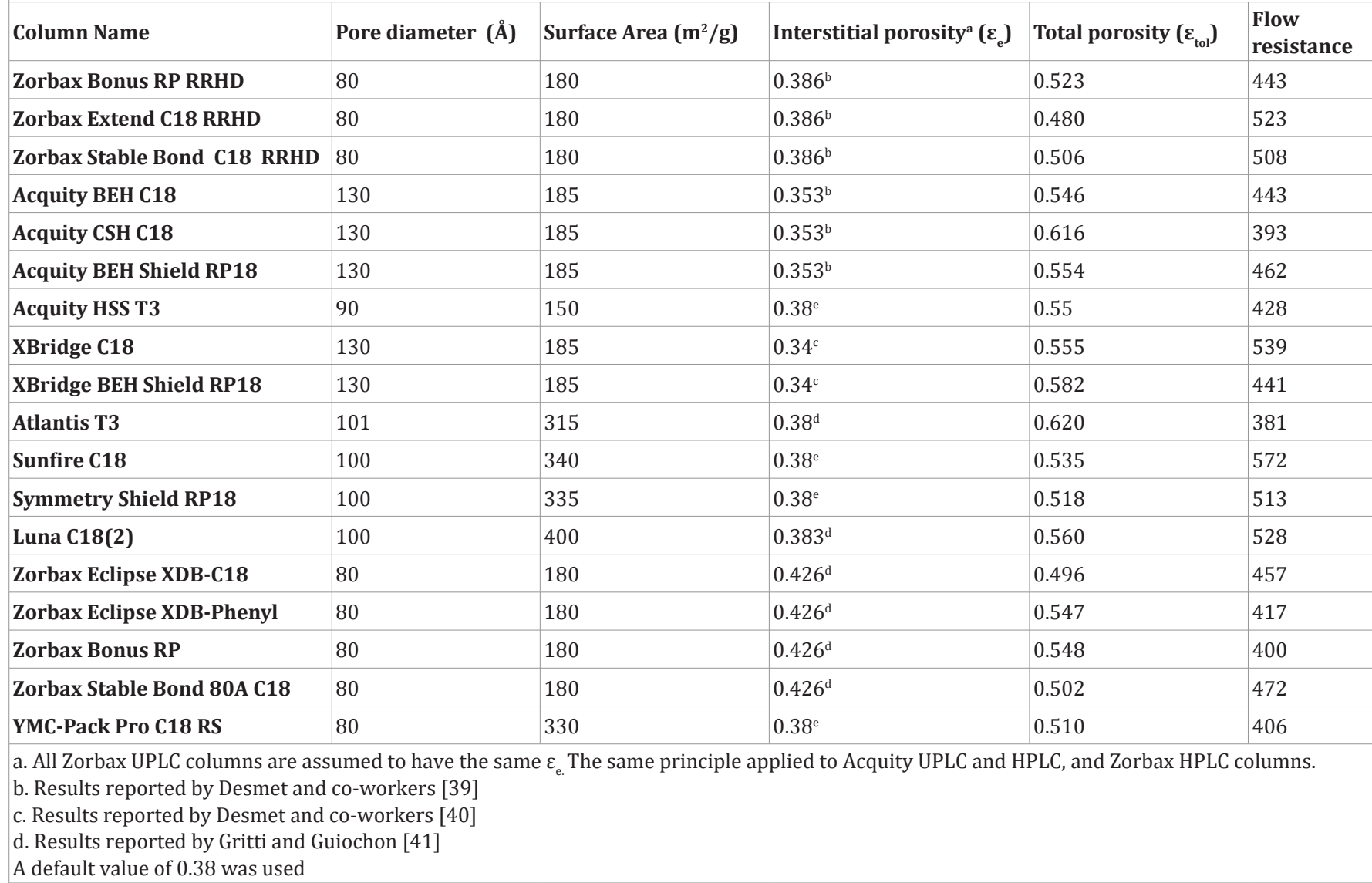

Table 4: Comparison of the retention time and peak width at half height obtained from simulation and experiments for HPLC system.

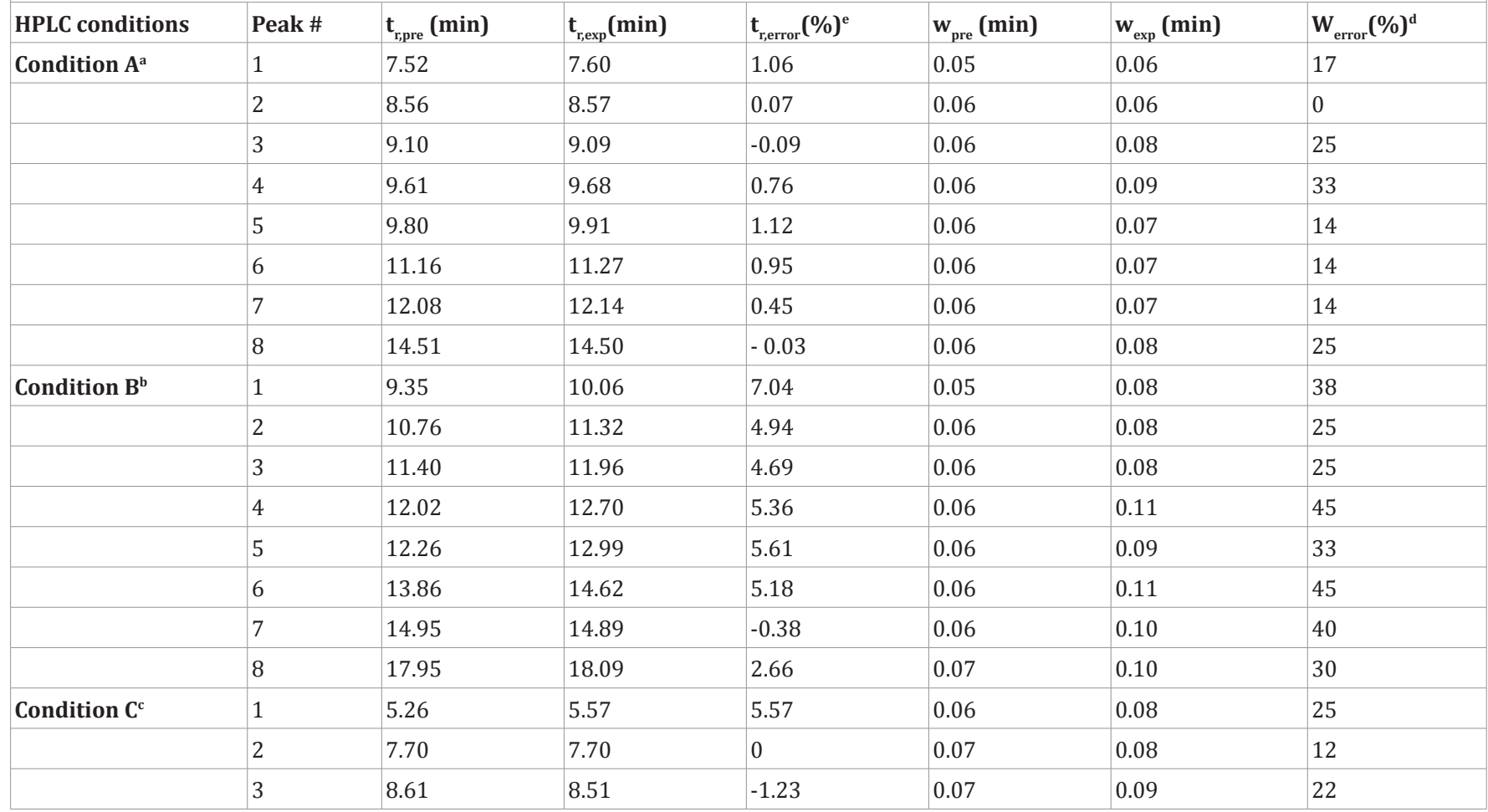




\begin{tabular}{|l|l|l|l|l|l|l|l|l|}
\hline & 4 & 9.63 & 9.63 & 0.04 & 0.07 & 0.14 & 50 \\
\hline & 5 & 10.04 & 10.07 & 0.29 & 0.08 & 0.10 & 20 \\
\hline & 6 & 12.72 & 12.67 & -0.43 & 0.08 & 0.09 & 11 \\
\hline & 7 & 14.72 & 14.54 & -1.26 & 0.09 & 0.10 & 10 \\
\hline & 8 & 20.47 & 19.98 & -2.46 & 0.10 & 0.11 & 9 \\
\hline
\end{tabular}

a. $\quad$ Gradient conditions: B\% increased from 20 to $64 \%$ linearly in $15 \mathrm{~min}$; column length: $25 \mathrm{~cm} ; \mathrm{F}=1.3 \mathrm{~mL} / \mathrm{min}$.

b. $\quad$ Gradient conditions: B\% increased from 20 to $62 \%$ linearly in $20 \mathrm{~min}$; column length: $25 \mathrm{~cm}$; F = $1.1 \mathrm{~mL} / \mathrm{min}$.

c. Gradient conditions: $\mathrm{B} \%$ increased from 33 to $53 \%$ linearly in $20 \mathrm{~min}$; column length: $40 \mathrm{~cm}$; F = $1.0 \mathrm{~mL} / \mathrm{min}$.

d. $\quad \mathrm{W}_{\text {error }}(\%)=\left|\mathrm{w}_{\text {pre }}-\mathrm{w}_{\text {exp }}\right| / \mathrm{w}_{\text {exp }} \times 100 \%$

e. $\quad t_{\text {rerror }}(\%)=\left(t_{\text {rpre }}-t_{\text {rexp }}\right) / t_{\text {rexp }} \times 100 \%$

All the gradient runs were conducted using XBridgeC18 columns packed with $3.5 \mu \mathrm{m}$ particles with the pore size of $130 \AA$. . $0.1 \%$ TFA in water was as solvent $A$ and acetonitrile was used as solvent B. The column temperature was fixed at $35^{\circ} \mathrm{C}$. Detection wavelength was set at $260 \mathrm{~nm}$.

Table 5: Comparison of the retention time and peak width at half height obtained from simulation and experiments for UPLC system.

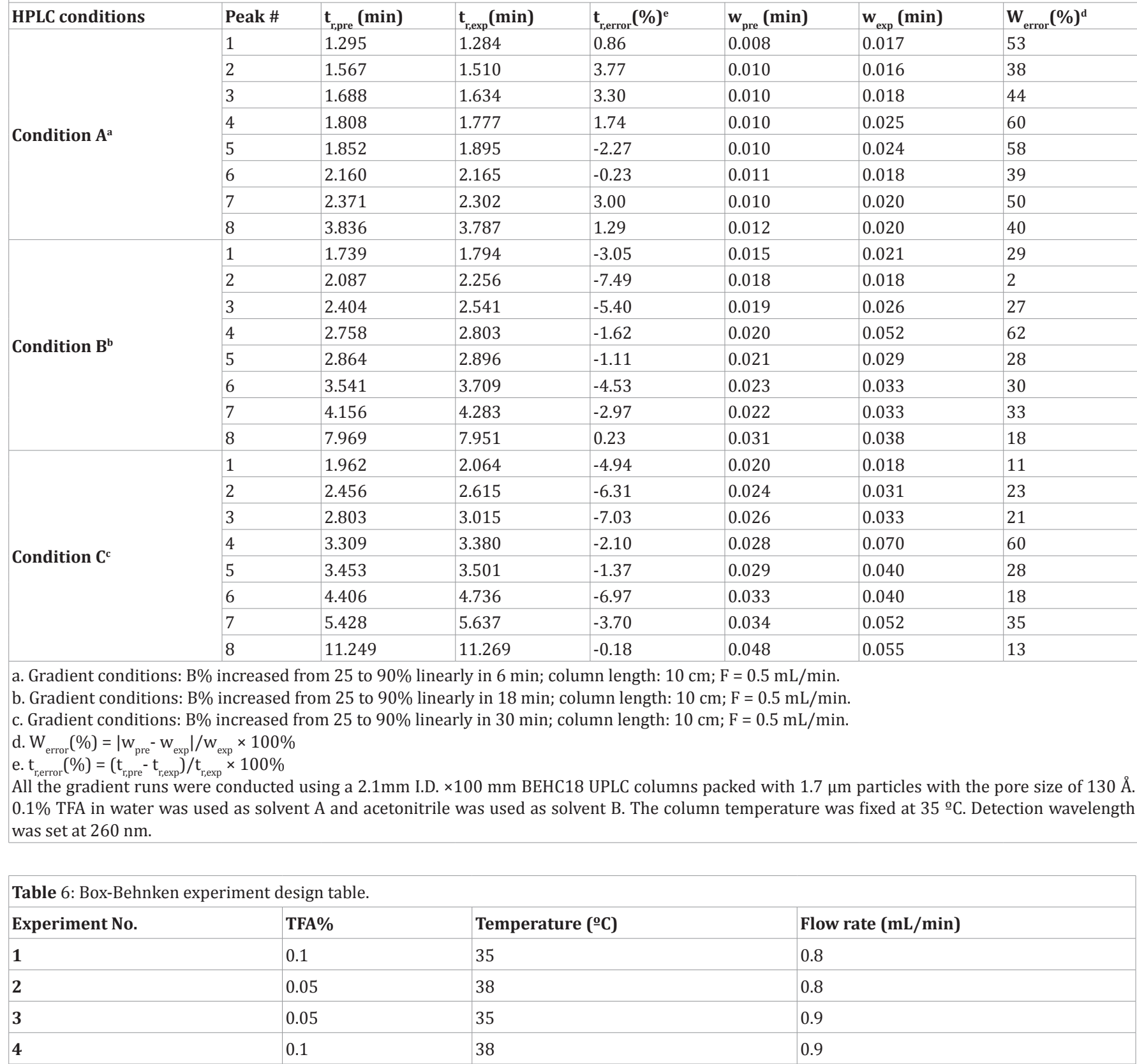




\begin{tabular}{|c|c|c|c|}
\hline 5 & 0.15 & 35 & 0.7 \\
\hline 6 & 0.1 & 38 & 0.7 \\
\hline 7 & 0.1 & 32 & 0.7 \\
\hline 8 & 0.15 & 32 & 0.8 \\
\hline 9 & 0.1 & 32 & 0.9 \\
\hline 10 & 0.15 & 38 & 0.8 \\
\hline 11 & 0.05 & 32 & 0.8 \\
\hline 12 & 0.1 & 35 & 0.8 \\
\hline 13 & 0.1 & 35 & 0.8 \\
\hline 14 & 0.15 & 35 & 0.9 \\
\hline 15 & 0.05 & 35 & 0.7 \\
\hline
\end{tabular}

For all the 15 HPLC experimental runs listed in (Table 6), experiments were operated on a $40 \mathrm{~cm}$ long column obtained by coupling a $25 \mathrm{~cm}$ XBridge C18 with another $15 \mathrm{~cm}$ XBridge C18 columns. Both of these columns were packed with $3.5 \mu \mathrm{m}$ particles with the pore size of $130 \AA$. The I.D. of both columns was $2.1 \mathrm{~mm}$. Detection wavelength was set at $260 \mathrm{~nm}$. For all these experiments, solvent A was either $0.05 \%, 0.1 \%$ or $0.15 \%$ TFA in water as defined by the TFA\% in (Table 6) for each experiment, and solvent B was acetonitrile. The B\% was increased from $33 \%$ to $53 \%$ linearly in 35 minutes. The sample injection volume was $5 \mu \mathrm{L}$ for all the experiments.

Table 7: Linear regression models for Critical Quality Attributes (CQAs).

\begin{tabular}{|c|c|c|c|c|c|}
\hline Quality Attribute & Term & Estimate & Standard Error & t Ratio & Prob $>|t|$ \\
\hline \multirow{3}{*}{ API peak tailing } & intercept & 1.50 & 0.005 & 281.20 & $<10^{-8}$ \\
\hline & TFA\% $(0.05,0.15)$ & -0.19 & 0.005 & -37.61 & $<10^{-8}$ \\
\hline & TFA $\% \times$ TFA $\%$ & 0.09 & 0.007 & 12.19 & $<10^{-8}$ \\
\hline \multirow{6}{*}{$t_{\mathrm{r}, \mathrm{API}}$} & intercept & 16.50 & 0.054 & 305.07 & $<10^{-8}$ \\
\hline & TFA\% $(0.05,0.15)$ & 0.56 & 0.04 & 14.00 & $2 \times 10^{-7}$ \\
\hline & temperature $(32,38)$ & -0.19 & 0.040 & -4.85 & $9.1 \times 10^{-7}$ \\
\hline & flow rate $(0.7,0.9)$ & -1.40 & 0.040 & -35.27 & $<10^{-8}$ \\
\hline & TFA $\% \times$ TFA $\%$ & -0.54 & 0.058 & -9.21 & $7.1 \times 10^{-6}$ \\
\hline & flow rate $\times$ flow rate & 0.20 & 0.058 & 3.34 & 0.0086 \\
\hline \multirow{5}{*}{$t_{\mathrm{r}, \text { imp4 }}$} & intercept & 15.83 & 0.051 & 312.50 & $<10^{-8}$ \\
\hline & temperature $(32,38)$ & -0.16 & 0.037 & -4.30 & 0.002 \\
\hline & flow rate $(0.7,0.9)$ & -1.37 & 0.037 & -36.78 & $<10^{-8}$ \\
\hline & TFA $\% \times$ TFA $\%$ & -0.51 & 0.055 & -9.41 & $5.9 \times 10^{-6}$ \\
\hline & flow rate $\times$ flow rate & 0.19 & 0.055 & 3.39 & 0.0079 \\
\hline \multirow{6}{*}{ Resolution $\mathbf{1}^{\mathrm{a}}$} & intercept & 2.20 & 0.020 & 108.71 & $<10^{-8}$ \\
\hline & TFA\% $(0.05,0.15)$ & 0.14 & 0.015 & 9.39 & $6 \times 10^{-6}$ \\
\hline & temperature $(32,38)$ & -0.03 & 0.015 & -1.84 & 0.098 \\
\hline & TFA $\% \times$ temperature & -0.08 & 0.021 & -3.67 & 0.0051 \\
\hline & TFA $\% \times$ TFA $\%$ & -0.21 & 0.022 & -9.38 & $6 \times 10^{-6}$ \\
\hline & temperature $\times$ temperature & -0.10 & 0.022 & -4.36 & 0.0018 \\
\hline \multirow{2}{*}{ Resolution $2^{\text {b }}$} & intercept & 3.75 & 0.081 & 46.25 & $<10^{-8}$ \\
\hline & flow rate $(0.7,0.9)$ & 0.29 & 0.111 & 2.61 & 0.023 \\
\hline
\end{tabular}


work is the first in which kinetic parameters of so many different columns are characterized and integrated in software for method development and optimization. In addition, since these columns are widely used in pharmaceutical industry, these column parameters also provide useful information for other HPLC/ UPLC method development software tools and applications.

\section{Mobile phase composition and column screening}

The API molecule is a basic compound containing several ring structures and functional groups that can interact with the silanol groups on columns via hydrogen bonding interactions. When $0.1 \%$ FA was used in mobile phases, severe peak tailing was observed. Since it is well known that ion pairing reagents can improve the peak shape for basic drugs [44-46], 0.1\% TFA was added to the mobile phases, which greatly improved the peak shape. In addition to using ion pairing reagents, another commonly used strategy to improve peak shape for basic compounds is to use basic mobile phases [47]. Therefore, mobile phases consisting of $\mathrm{MeOH}-0.1 \% \mathrm{NH}_{4} \mathrm{OH}$ aqueous and ACN$0.1 \% \mathrm{NH}_{4} \mathrm{OH}$ aqueous were screened. These basic mobile phase conditions were only used on columns that were compatible with basic mobile phases.

Five UPLC/UHPLC columns were selected for column and mobile phase composition screening. These five columns included three C18 columns, namely Zorbax SB C18, Zorbax Extend C18, and Acquity BEH C18. Zorbax SB C18 column was selected due to its extremely good stability under acidic mobile phase conditions. Water BEH C18 was selected due to its unique stability in both acidic and basic mobile phases. Extend C18 column was selected mainly to test the peak shape improvement of API under basic mobile phase conditions. In addition, two Polar Embedded Group (PEG) columns, Zobax RP bonus and Acquity BEH Shield RP18 columns were selected due to their unique selectivity that was very different from the selectivity of C18 columns [48].

Another challenge for this method development was the separation of API and impurity 4 (see Figure 1 and Figure 5). LC-MS results showed that these two molecules had very similar structures. The only structural difference between these two molecules was an extra double bond in API molecule. Column screening results showed that under all the tested gradient scouting conditions, only the Acquity BEH C18 column partially resolved this critical pair using ACN-0.1\% TFA aqueous as the mobile phase. Using the ACN-0.1\% TFA aqueous mobile phase, the Zorbax Extend C18 only produced marginally partial separation. For all the other columns and mobile phase conditions, no separation of this critical pair was observed. Therefore, retention times of the API and the seven chosen impurities obtained from the three gradient scouting runs on the Acquity BEH C18 column using ACN- 0.1\% TFA aqueous mobile phase were used in the next step for further optimization.

\section{Gradient elution simulation and optimization}

The theory for gradient elution simulation and optimization have been discussed and described in detail [12-14, 19,21,23,25]. In addition, simultaneous optimization of operational parameters including flow rate, column length and mobile phase gradient profile at given gradient times using Microsoft Excel Solver function have been reported previously $[24,29,49]$. The same principles and procedures were used in this work. However, by integrating all the calculation logic, physical chemistry constants and various column parameters in the VBA code and MySQL database, the entire simulation and optimization operated automatically and with better prediction accuracy. In addition, the automation enables users to optimize the operational conditions without having to have in-depth knowledge and understanding of gradient theory and simulation. Finally, integration of interactive Graphic User Interfaces (GUI) makes the program user-friendly and easy to use. Figure 2 shows the user interface of the Excel simulation software that allows users to submit there tention times of API and impurities under the three gradient scouting run conditions, the operational conditions of the scouting runs, the gradient void volume of the instrument and dead volume of the column for the simulation. Figure 3 shows the GUI for users to set up the constraints for the optimization, including column format, organic modifier of the mobile phase, column temperature, gradient time and the maximum column pressure allowed for the optimization. After the user submits the constraints, the simulation program then proposes the optimum operational conditions. Figure 4 shows the simulated chromatogram under the proposed optimum operational conditions generated by the software. The GUI allows users to change operational conditions including organic modifier percentage of the gradient and flow rate. Once users change any of these conditions, the simulated chromatogram will be changed accordingly. This allows users to check how these operational conditions affect the separation.

The accuracy of the simulation program was studied by first separating the API mixture sample under three different gradient elution conditions, and then comparing the experimentally obtained retention times and peak widths with the values predicted by the simulation program under the same conditions. Figure 5 shows three chromatograms obtained from experiments under the corresponding gradient elution conditions. (Table 4) shows the predicted and experimentally obtained retention times and peak widths at half height for API and seven chosen impurities under these operational conditions from table 4 . The accuracy of the predicted retention time is within $8 \%$. However, the experimentally measured peak widths are much broader than the predicted values. The extra peak broadening is very possibly due to the column overloading [45]. And the secondary interaction between API and the column. Such an extra peak broadening effect was not considered in the simulation program.

It should be noted that the simulation program used the retention times of API and representative impurities measured on an Acquity BEH C18 UPLC column using an UPLC instrument system, and predicted retention times and peak widths on an X Bridge C18 column operated on an HPLC system with good accuracy. Applying UPLC systems to the method development of conventional HPLC can greatly reduce the time and solvent consumption required for the method development.

An interesting question is if it is possible to predict the 


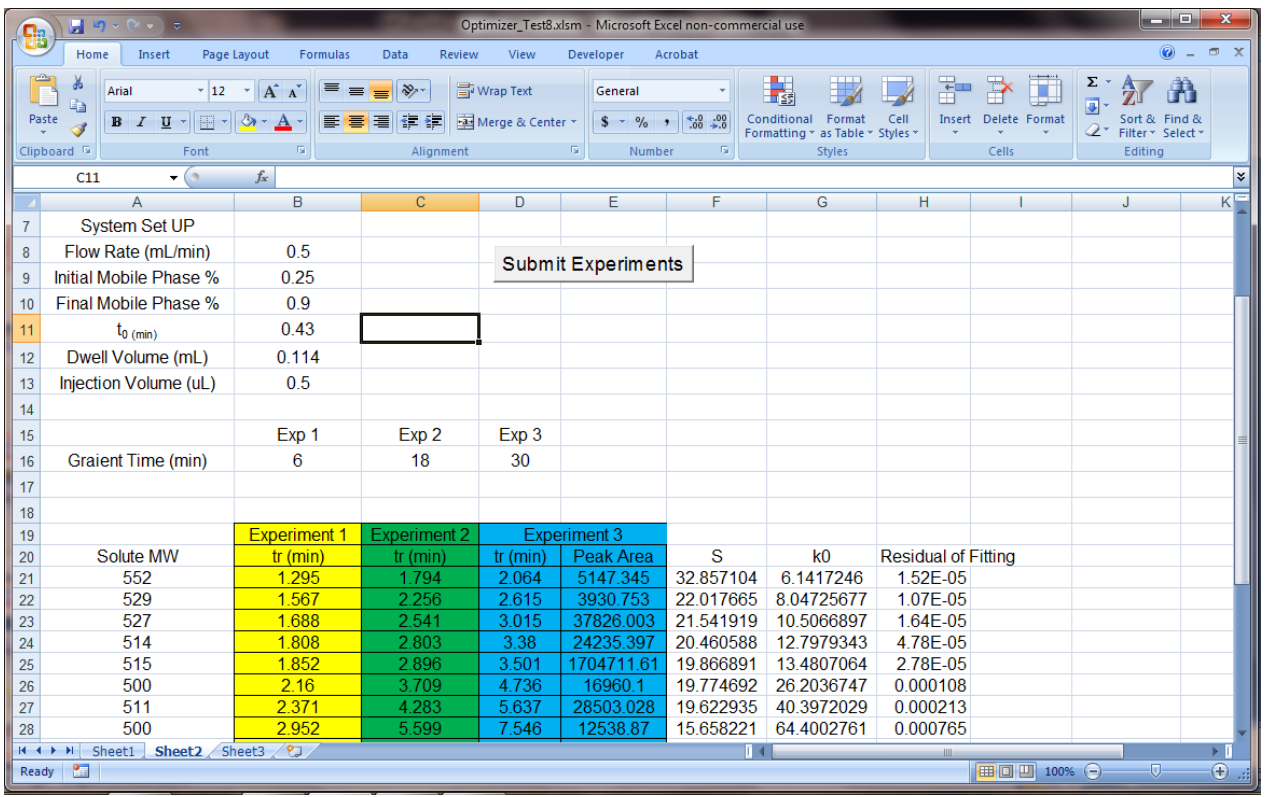

Figure 2: Graphic User Interface of the Excel VBA simulation program that allows users to submit the retention times of API and impurities obtained from the three gradient ramp runs, the operational conditions of the gradient ramps and the instrument configuration information.

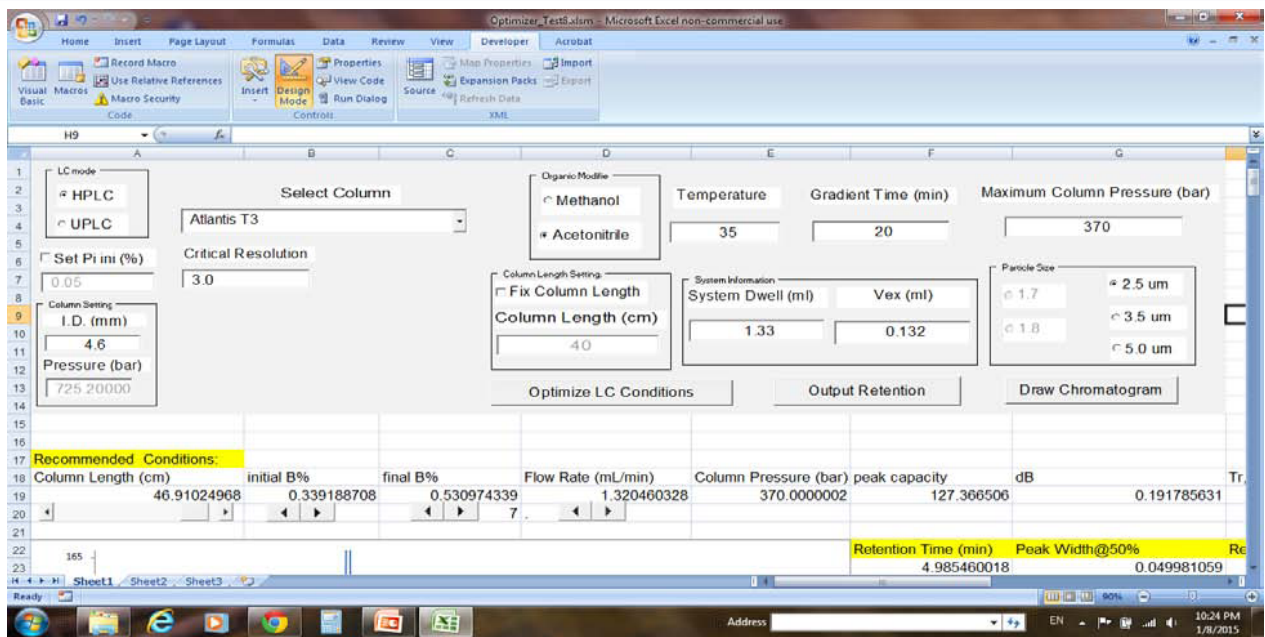

Figure 3: Graphic User Interface of the Excel VBA simulation program that allows users to set up the constraints for gradient condition optimization.

retention times and peak widths under given UPLC conditions using the retention times measured in HPLC conditions. To answer this question, based on the retention times of API and seven impurities measured under the three different HPLC gradient conditions listed in Figure 5. The retention times and the corresponding peak widths of these peaks under three different UPLC operational conditions were predicted by the simulation software, and compared to the experimentally measured values under the corresponding UPLC operational conditions. The results are listed in Table 5. From table 5, the accuracy of the predicted retention time is within $8 \%$, which is similar to the prediction results for HPLC shown in table 4. In terms of the peak width, similar to the HPLC prediction results, the experimentally measured peak widths are much broader than the predicted values for UPLC prediction results. It should be noted that due to the smaller column dimensions used in the UPLC experiments, compared to HPLC, the peak widths in UPLC experiments are more sensitive to the instrument configurations, especially the volume of connection tubing and valves. From the results shown in table 4,5 . The simulation software can predict the retention behavior and optimize the operational conditions for HPLC and UPLC systems based on the retention times measured under either HPLC or UPLC conditions, although the most efficient application in practice is to use the measurements obtained from UPLC systems to optimize the operational conditions for HPLC systems.

The gradient conditions in figure 5 . Were proposed by the simulation program as optimum conditions under different constraints $(25 \mathrm{~cm}$ maximum column length and 15 min gradient 


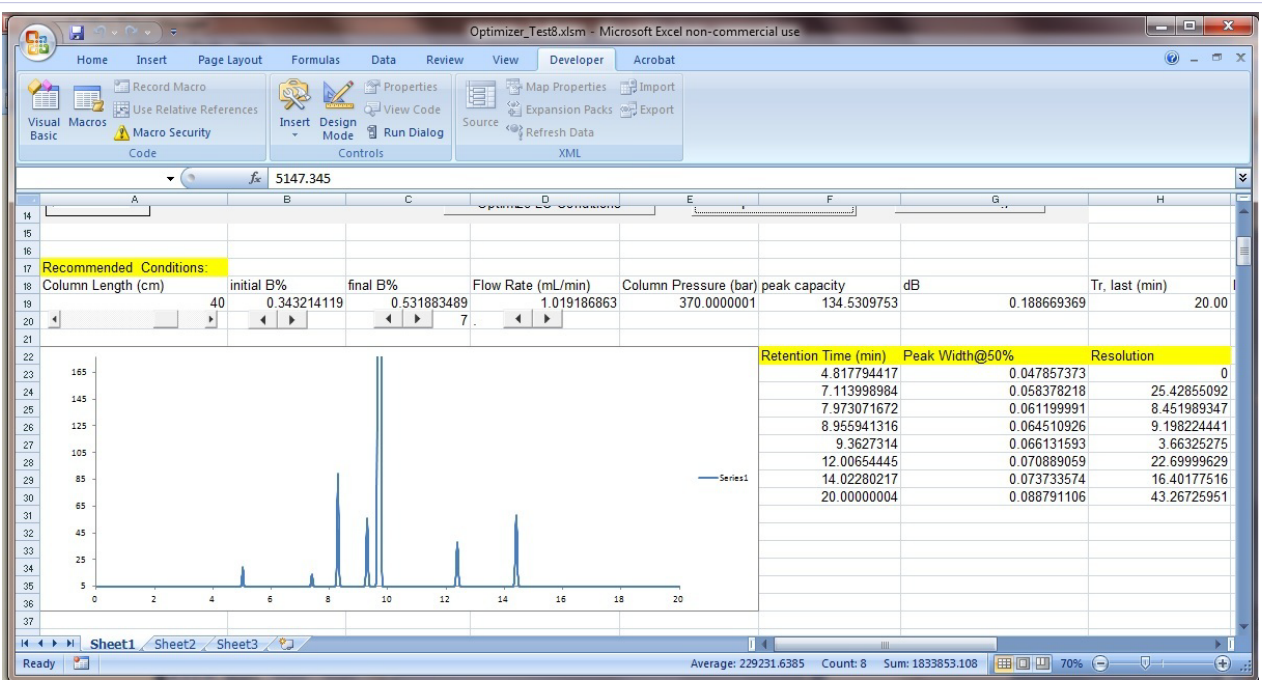

Figure 4: Chromatogram generated by the simulation program under the proposed optimum gradient conditions. By clicking increment and decrement buttons under column length, initial B\%, final B\% and flow rate labels, the chromatogram will change accordingly, which allows users to see the effects of these operational conditions on the separation.
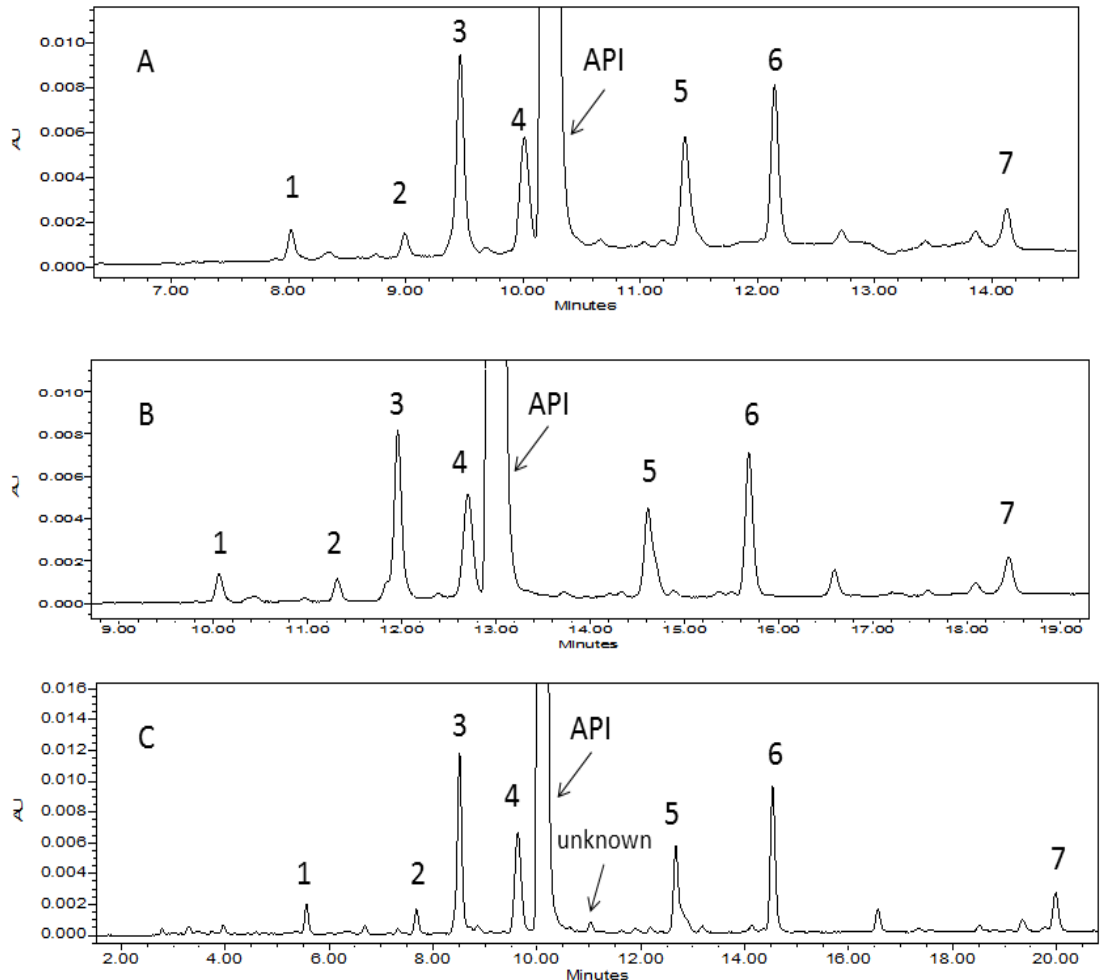

Figure 5: Chromatograms of API mixture obtained under three gradient conditions.

All the gradient runs were conducted using XBridgeC18 columns (2.1 mm I.D.) packed with $3.5 \mu \mathrm{m}$ particles and a pore size of $130 \AA . .0 .1 \%$ TFA in water was used as solvent $A$ and acetonitrile was used as solvent B. The column temperature was fixed at $35{ }^{\circ} \mathrm{C}$. Detection wavelength was set at $260 \mathrm{~nm}$. Figure 5A gradient conditions: $\mathrm{B} \%$ increased from 20 to $64 \%$ in $15 \mathrm{~min}$; column length: $25 \mathrm{~cm} ; \mathrm{F}=1.3 \mathrm{~mL} / \mathrm{min}$. The resolution of API and impurity 4 in this figure was 1.6.

Figure 5B gradient conditions: $B \%$ increased from 20 to $62 \%$ in $20 \mathrm{~min}$; column length: $25 \mathrm{~cm} ; \mathrm{F}=1.1 \mathrm{~mL} / \mathrm{min}$. The resolution of API and impurity 4 in this figure was 1.6.

Figure 5C gradient conditions: B\% increased from 33 to 53\% in $20 \mathrm{~min}$; column length: $40 \mathrm{~cm}$ (obtained by coupling a $25 \mathrm{~cm}$ and another $15 \mathrm{~cm}$ columns); $F=1.0 \mathrm{~mL} / \mathrm{min}$. The resolution of API and impurity 4 in this figure was 2.1 . 
time for figure 5A; $25 \mathrm{~cm}$ maximum column length and $20 \mathrm{~min}$ gradient time for figure $5 \mathrm{~B}$; and $50 \mathrm{~cm}$ maximum column length and 20 min gradient time for figure $5 \mathrm{C}$. The maximum column pressure constraint was set at 350 bar for all these simulations. According to figure 5 resolution between API and impurity 4 had already achieved the criterion of 2.0 for the operational conditions of figure 5 . However, considering that the quality control team preferred to conduct analyses at column pressures lower than 300 bars during the method transfer, the operational conditions described in figure $5 \mathrm{C}$ were modified as follows: the flow rate was decreased from $1.0 \mathrm{~mL} / \mathrm{min}$ to $0.8 \mathrm{~mL} / \mathrm{min}$ and gradient time was increased from $20 \mathrm{~min}$ to $35 \mathrm{~min}$. The resulting conditions were: $\mathrm{B} \%$ increased from 33 to $53 \%$ in $35 \mathrm{~min}$; column length: $40 \mathrm{~cm} ; \mathrm{F}=0.8 \mathrm{~mL} / \mathrm{min}$. Under the modified conditions, the resolution between API and impurity 4 was modestly improved to 2.3. The modified operational conditions were then used for DOE study.

It should be noted that although changing the column temperature has been reported as one of the most effective ways to optimize the resolution and peak capacity of gradient elution[23,24,35,50], and optimizing the column temperature using simulation programs has been successfully demonstrated $[13,14,23,24,50]$, the column temperature was not optimized by the simulation program in this work. The column temperature was fixed at $35^{\circ} \mathrm{C}$ for all column screening experiments and the following simulations and optimization experiments in order to prevent degradation of process impurities and the API that might occur at higher column temperatures. Since the optimum operational condition obtained from the simulation program had already achieved the resolution criteria, exploring higher column temperatures was not considered in this step. In the next step, surface response design was used to explore a narrow column temperature range of $30-40{ }^{\circ} \mathrm{C}$ to define the operable region.

\section{Selection of DOE Factors and DOE techniques}

Three operational variables were selected to be further studied by DOE based on the optimized operational conditions obtained from the previous steps. The first factor selected was TFA\%. As an ion-pair reagent, it is well known that TFA\% affects both peak tailing and selectivity of basic compounds [44-46]. Since both of these factors played a critical role in resolution, a complicated effect of TFA\% on resolution and peak shape was expected. The second factor was temperature effects. Temperature affects resolution by affecting selectivity and peak width [24]. In addition, since temperature also affects the ion pairing process, it should have a complicated effect on resolution and API peak shape as well. The third factor studied was flow rate. Flow rate affects resolution and peak capacity by affecting gradient steepness and separation efficiency [24,28]. In addition, in the practice of sample analysis, flow rate usually can be adjusted to compensate the shift of API retention time caused by different instrument configurations. Therefore, studying how the change in flow rate affects CQAs is critical. Although the effects of other operational parameters, such as sample concentration, sample injection volume and initial and final organic modifier percentages in mobile phase have been studied by DOE [4]. These factors were not studied in this work because these parameters can be very well controlled by careful sample preparation and the high quality of the modern chromatography instrument systems.

A: API retention

B: API peak tailing
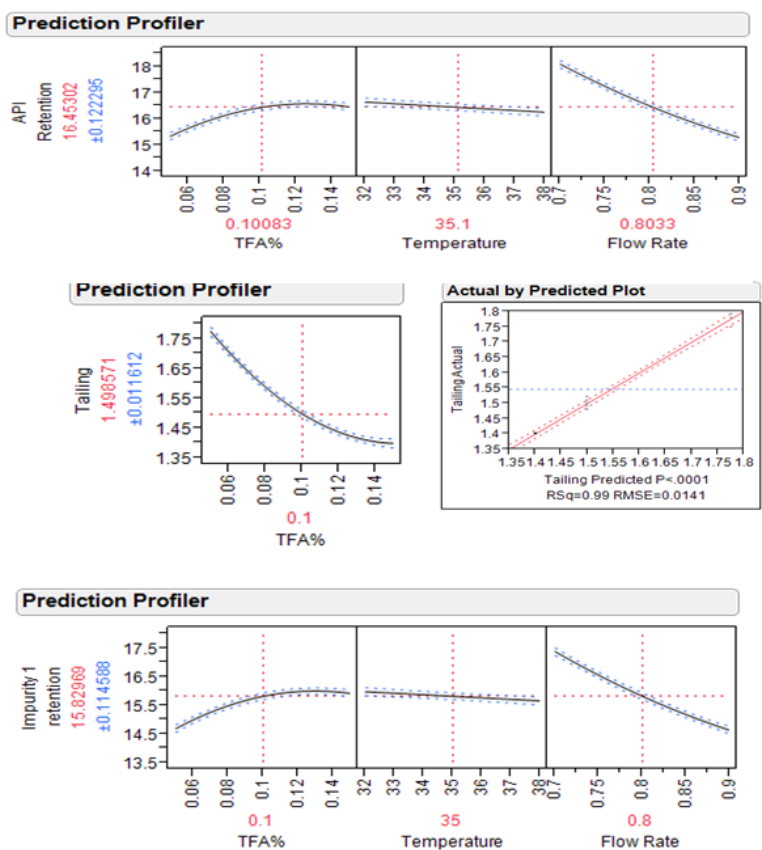

Figure 6: API tailing factor and retention and impurity 4 retention versus the operational conditions based on the regression model established by JMP. 
Since operational parameters have already been optimized by the previous steps and all the CQA criteria have been fulfilled, the purpose of this step was to accurately approximate the true response function within a relatively small region near the optimized conditions obtained from the previous steps. In addition, since flow rate, column temperature and TFA $\%$ affected the resolution and API peak shape in a complicated way, nonlinear effects of these factors on CQAs were expected. Therefore, a surface response design that can fit nonlinear models should be used [7]. The Box-Behnken design was selected because it is a surface response method that can model nonlinear effects, and is very efficient when3-4 factors are involved in the model [30].

\section{Effects of operational parameters on API retention time}

The linear regression model of API retention time versus operational parameters is shown in Table7. Plots generated by JMP software based on this model are shown in figure $6 \mathrm{~A}$. According to figure 6A. API retention time increases with the increase of TFA\%. In addition, the increase in API retention becomes lower when the TFA\% was higher than a certain value $(\sim 0.1 \%$ in figure $6 \mathrm{~A})$, and finally asymptotically approaches a limit. This trend is consistent with the general trend of basic analyte retention observed previously when TFA is used in the mobile phase [51]. According to Dai [44]. The retention of basic analytes at low $\mathrm{pH}$ mobile phase conditions is the consequence of two processes: one is the retention of the neutral ion pairs formed between the basic analytes and ion pair reagents. The other is the ion exchange between the analyte cations and the anionic ion pair reagents adsorbed on the stationary phase. Applying this mechanism, when TFA\% is low, with the increase of the TFA\%, more ion pairs are formed between API and TFA, which increases the retention time of API. In addition, with the increase of TFA\%, more anionic TFA will adsorb onto the column. As a result, more API cations will be retained on the column by interacting with the anionic TFA adsorbed on the column. Therefore, for both ion pairing and dynamic ion exchange processes, increasing TFA concentration will increase the retention time of API. However, based on both ion paring and dynamic ion exchange mechanisms, retention time of API is not a linear function of the TFA concentration. To explain this, it will be useful to review the retention model proposed by Kazakevich [52]. Although this model only considers the ion pairing process, the same principles can be applied to dynamic ion exchange process.

According to Kzakevich model, the retention factor of analyte is defined by the following equation:

$$
k=k_{f} \theta+k_{i p}(1-\theta)=k_{i p}-\theta\left(k_{i p}-k_{f}\right)
$$

In this equation, $\mathrm{k}$ is the retention factor of the analyte, $\mathrm{k}_{\mathrm{f}}$ and $\mathrm{k}_{\mathrm{ip}}$ are the retention factors of the free and ion paired forms of the analyte, respectively, and $\theta$ is the fraction of the free form of the analyte. Since both $\mathrm{k}_{\mathrm{f}}$ and $\mathrm{k}_{\mathrm{ip}}$ are constants, we can define another constant $\mathrm{k}^{\prime}=\mathrm{k}_{\mathrm{ip}}-\mathrm{k}_{\mathrm{f}}$. Considering that fact that the free analyte cations usually are less retentive than the neutral and more hydrophobic, ion-paired analyte species on reversed-phase columns, $\mathrm{k}_{\mathrm{f}}$ is usually smaller than $\mathrm{k}_{\mathrm{ip}}$. Therefore, $\mathrm{k}^{\prime}>0$.
A
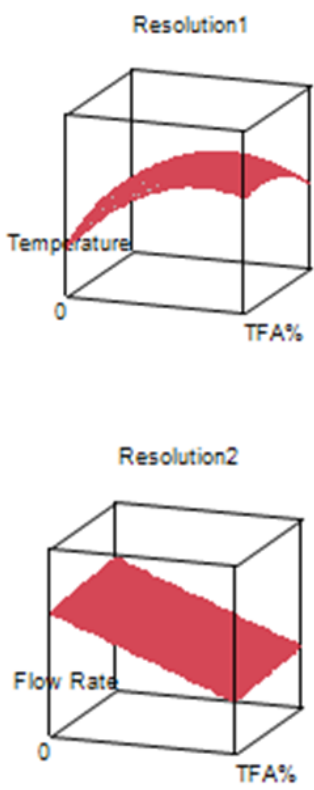
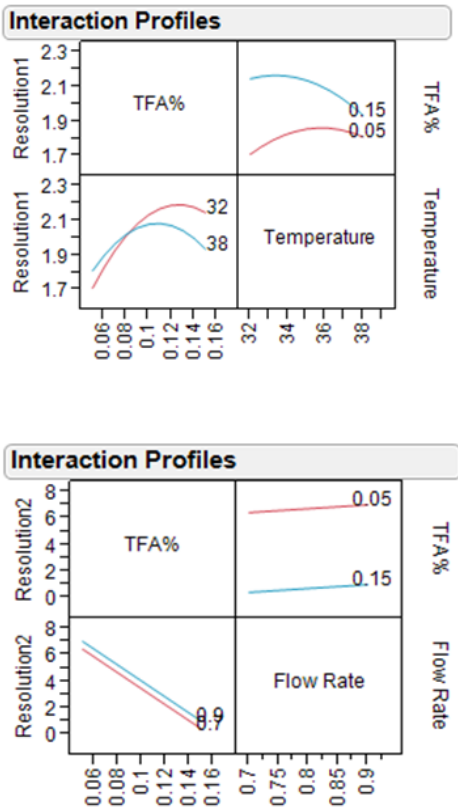

Figure 7: Resolutions between API and impurity 4 and unknown impurity as function of operational parameters. The plots were generated by JMP 9.0 software. 
Considering the ion pairing equilibration, $\theta$ can be expressed as

$$
\theta=\frac{1}{K\left[A^{-}\right]+1}
$$

In Eq. $2, \mathrm{~K}$ is the equilibrium constant of the ion pairing process, and $\left.\mathrm{A}^{-}\right]$is the concentration of the ion pairing reagent. Submitting $\theta$ in Eq. 2 to Eq. 1, we get

$$
k=k_{i p}-\frac{k_{i p}-k_{f}}{K\left[A^{-}\right]+1}=k_{i p}-\frac{k^{\prime}}{K\left[A^{-}\right]+1}
$$

In Eq. 3, $\mathrm{k}_{\mathrm{ip}}$ can be considered as the "limiting retention factor" for ion paired analyte, which corresponds to the retention factor of fully ion paired analyte at very high concentration of ion pairing reagent [52]. Since the second item in Eq. 3 is positive, and it asymptotically approaches to zero with the increase of $\left[\mathrm{A}^{-}\right]$, increasing ion pairing reagent concentration leads to the asymptotic approach of the analyte retention to $\mathrm{k}_{\mathrm{ip}}$, which is consistent to the nonlinear trend observed in figure $6 \mathrm{~A}$.

Although Kzakevich model only considers the ion pairing equilibration in the system, the dynamic ion exchange process can be described by the same format as expressed by Equation 3 [44]. As a result, with the increase of TFA concentration in the mobile phase, the retention factor of the analyte governed by the dynamic ion exchange process will finally asymptotically approaches the limiting retention factor corresponding to the maximum adsorption of TFA anions on the column. Since retention factors governed by ion pairing and dynamic ion exchange processes both asymptotically approach their maximum values with the increase of TFA concentration, it is not surprising to see the nonlinear trend of retention time versus the TFA concentration in figure $6 \mathrm{~A}$.

It should be noted that the regression model listed in table 7 was used to approximate API retention versus operational parameters in a narrow region near the optimized operational conditions. It does not mean that the relationship between the retention and TFA concentration is fundamentally governed by a second order polynomial model. In fact, according to the fundamental separation mechanisms, the retention factor of basic compounds is a complicated function of the ion pair reagent concentration $[44,53]$. In addition, from figure $6 \mathrm{~A}$, increasing flow rate greatly reduces the retention time, while temperature only slightly affects the retention time.

\section{Effects of operational parameters on API peak tailing factor}

Table 7 showed the linear regression model of peak tailing factor versus operational parameters. The plots generated by JMP software based on the model are shown in figure. 6B. Shows that when TFA\% is lower than $0.1 \%$, as TFA\% was increased, the peak tailing factor improves significantly from 1.75 to 1.5 . However, when TFA $\%$ is higher than $0.1 \%$, improvements in the peak tailing factor become slower with the further increases of TFA\%. This can be explained by the same mechanisms as in the API retention discussion. According to the study of Dai [45], peak tailing and peak broadening of basic compounds are caused by sample overloading, and increasing ion pairing reagent concentration improves the peak width and peak shape by increasing the amount of the basic analyte participating in both ion paring and dynamic ion exchange processes, which increases the sample loading capacity. From Eq.2, it is easy to see that the fraction of the analyte participating in the ion paring process, which is $1-\theta$, can be defined as:

$$
1-\theta=\left(K\left[A^{-}\right]\right) /\left(K\left[A^{-}\right]+1\right)_{\mathrm{Eq}} .
$$

From Equation 4, in the low TFA concentration region where $K\left[A^{-}\right]<<1,1-\theta$ will increase with $\left[A^{-}\right]$fast, leading to great improvement in the peak width and peak shape. However, with the increase of TFA concentration, when $\left.\mathrm{K}^{-} \mathrm{A}^{-}\right]$is comparable to $1,1-\theta$ will increase with TFA concentration slower. With the further increase of the TFA concentration when $K\left[\mathrm{~A}^{-}\right]>1,1-\theta$ will approach to 1 asymptotically. As a result, increasing TFA concentration in high TFA concentration region will lead to a smaller increase in the fraction of the basic analyte participating in ion pairing, and therefore, lead to smaller improvement in peak shape compared to in the low TFA concentration region. The same principle applies to dynamic ion exchange process

\section{Effects of operational parameters on impurity 4 retention time}

The linear regression model of impurity 4retention time versus operational parameters is shown in Table7. Although the retention time of impurity 4 is not a CQA, understanding how the operational parameters affect the retention of this impurity will give us more insights into the mechanism of the separation between API and this impurity.

Plots generated by JMP software based on the linear regression model are shown in figure $6 \mathrm{C}$. Comparing (Figures $6 \mathrm{~A}$ and 6C). It is obvious that the retention times of API and impurity 4 show the similar nonlinear trend versus TFA\%, which implies that similar to API, impurity 4 also interacts ion paired with TFA. In addition, similar to the retention time of API, the retention time of impurity 4 is affected by both temperature and flow rate.

\section{Effects of operational parameters on resolution between API and impurity 4}

The linear regression model of the resolution between API and impurity 4 (defined as resolution 1) versus operational parameters is shown in Table7. Plots generated by JMP software based on this model are shown in figure7 A.

Figure 7A shows that resolution 1changes with TFA\% in a nonlinear way, with the optimum TFA $\%$ at $\sim 0.1 \%$. As shown in the previous sections, both the retention times and peak widths of API and impurity 4 change with TFA\% nonlinearly. Therefore, it is not surprising to observe a complicated, nonlinear effect of $\mathrm{TFA} \%$ on the resolution between them.

From table 7 , It is interesting to see that resolution 1 is affected by temperature $\times \mathrm{TFA} \%$ and temperature $\times$ temperature, 
but not by temperature itself (prob $>|t|>0.05)$. In addition, the interaction profiles in figure 7A Show strong interaction effects between TFA $\%$ and temperature, indicating that temperature affects resolution 1 by affecting the interactions between TFA and the basic analytes (API and impurity 4).

\section{Effects of operational parameters on resolution between API and an unknown impurity}

During the method development, an unknown impurity, which was eluted behind API was observed (see Figure. 5C) Analyzing the effects of operational parameters on the retention of this impurity (results not shown here) showed that TFA\% has no effects on the retention of this impurity. Therefore, this impurity does not interact with TFA by ion pair or ion exchange.

The linear regression model of the resolution between API and this impurity (defined as resolution 2) versus operational parameters is shown in table 7. Plots generated by JMP software based on this model are shown in figure 7B. Figure 7B Showed that the resolution between API and this impurity decreases with the increase of TFA\%. This can be explained by the different interactions between API and the impurity with TFA. As a consequence of interacting with TFA by ion pairing and ion exchange, the retention time of API increases with the increase of TFA\%. However, the retention time of the impurity does not change with the increase of TFA\% because it does not interact with TFA. Since the impurity is eluted after API, with the increase of TFA\%, the API peak will move closer to the impurity peak, leading to a continuous decrease of the resolution between them. In addition, the interaction profiles of resolution 2 (right hand side of figure 7B show parallel lines corresponding to resolution 2 versus flow rate when TFA $\%$ are $0.15 \%$ and $0.05 \%$, respectively. The same parallel lines are observed for resolution 2 versus TFA\% when flow rates are 0.7 and $0.9 \mathrm{~mL} / \mathrm{min}$, respectively. These parallel lines indicate that no interactive effects exist between flow rate and TFA\%. This is consistent to the fact that fundamentally flow rate should not affect ion pairing and ion exchange processes.

\section{Defining the Method Operable Design Region (MODR)}

The MODR was defined by the contour plots generated by JMP software based on the regression models that described how operational conditions affected CQAs. These contour plots were generated by setting the following thresholds: resolution $1>2.0$, resolution $2>2.0$ and API peak tailing $\leq 1.5$. The resulting contour plots are shown in figure 8 . According to these contour plots, to fulfill the criteria for resolution 1 , a safe region can be defined as $0.08 \%<\mathrm{TFA} \%<0.14 \%$ and $32{ }^{\circ} \mathrm{C}<$ column temperature $<38$ ${ }^{\circ} \mathrm{C}$. Similarly, to satisfy resolution 2 requirements, the region is
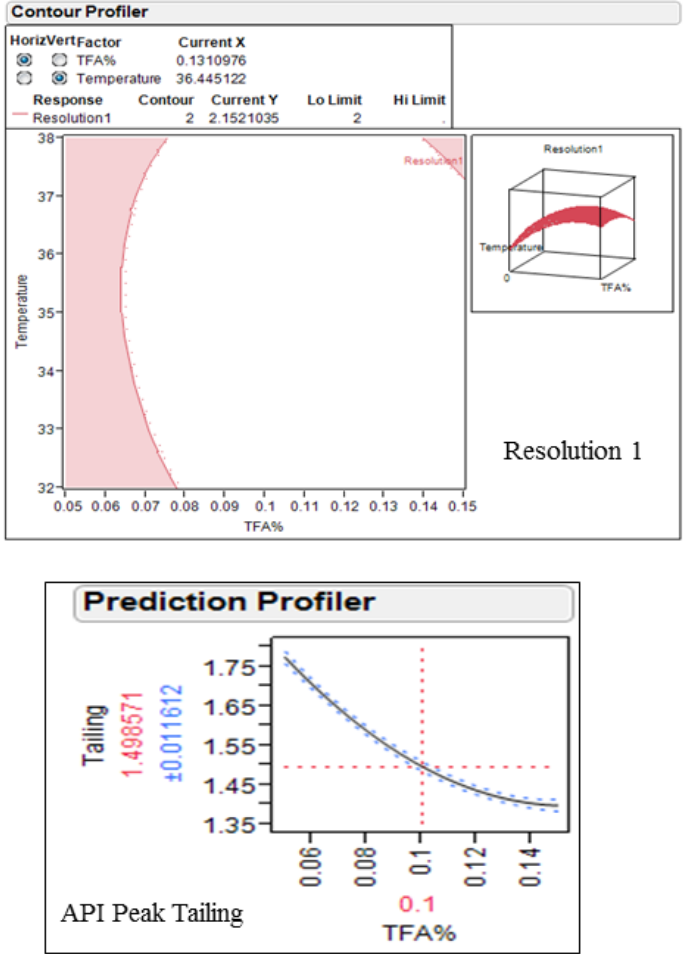

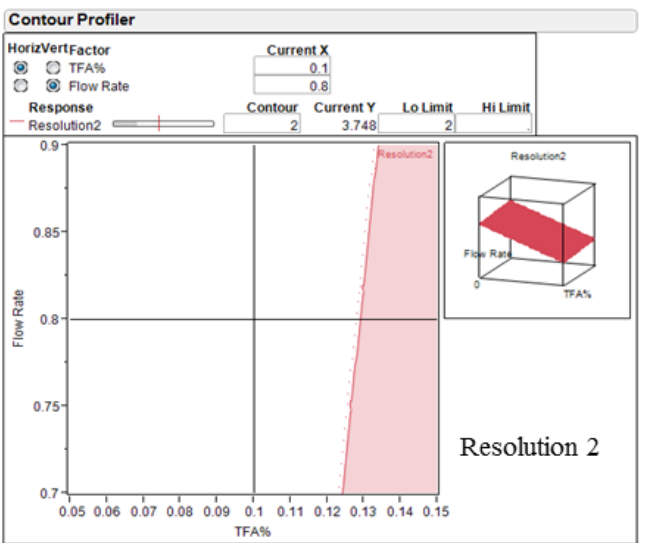

Figure 8: Contour plots for resolution 1, resolution 2 and prediction profile for API tailing factor

Resolution 1 is the resolution between API and impurity 4 (see Figure. 5)

Resolution 2 is the resolution between API and unknown impurity (see Figure. 5C)

The settings for the contour plots are: resolution $1>2.0$; resolution $2>2.0$. 
defined as $0.05 \%<\mathrm{TFA} \%<0.125 \%$ and $0.7 \mathrm{~mL} / \mathrm{min}<$ Flow rate $<0.9 \mathrm{~mL} / \mathrm{min}$. In addition, to satisfy the requirement of API peak tailing $\leq 1.5$, TFA $\%$ should be higher or equal to $0.1 \%$. Finally, by combining the above three regions, the MODR is defined as: $0.1 \%<\mathrm{TFA} \%<0.125 \%, 32{ }^{\circ} \mathrm{C}<$ column temperature $<38{ }^{\circ} \mathrm{C}$, and $0.7 \mathrm{~mL} / \mathrm{min}<$ Flow rate $<0.9 \mathrm{~mL} / \mathrm{min}$.

\section{Conclusion}

A framework integrating computer simulation and surface response design for reversed phase HPLC method development was applied to the method development of an API mixture sample. By applying the solvent retention parameters obtained from UPLC/UHPLC experiments to conventional HPLC method development, the time and solvent consumption required for HPLC method development were greatly reduced. In addition, an Excel simulation program coded by VBA was developed for automatic gradient operational condition optimization. This Excel program integrated the flow resistance and kinetic parameters of 18 commonly used UPLC/UHPLC and HPLC columns. In addition, integration of Graphic User Interfaces (GUI) makes it easy for users without in-depth understanding of gradient elution theory and programming to optimize the gradient conditions. Results showed that the simulation program predicted the retention times accurately and can propose the optimum operational conditions under given constraints. Using simulated chromatograms, the simulation program also allows users to fine-tune the operational conditions and see how the operational conditions affect the separation. A surface response method, BoxBehnken design was used to define the Method Operable Design Region (MODR) based on the optimized gradient conditions obtained from simulation and HPLC experiments. Finally, this work demonstrated that statistical analysis of DOE can provide further insights into the separation mechanisms, especially the effects of ion-pairing and ion-exchange processes on the CQAs of the HPLC method developed in this work.

\section{References}

1. International Conference on Harmonization (ICH). Q8(R2) Pharmaceutical Development. August 2009: https://www.pmda. go.jp/files/000156835.pdf

2. International Conference on Harmonization (ICH). Q9: Quality Risk Management. Novermber 2005: http://www.ich.org/fileadmin/ Public_Web_Site/ICH_Products/Guidelines/Quality/Q9/Step4/Q9_ Guideline.pdf

3. International Conference on Harmonization (ICH) Q11: Development and Manufacture of Drug Substances Draft Fuileline. May 2011:

http://www.ich.org/fileadmin/Public_Web_Site/ICH_Products / Guidelines/Quality/Q11/Q11_Step_4.pdf

4. Reid GL, Cheng G, Fortin DT, Harwood JW, Morgado JE, Wang J, et al. Reversed-phase liquid chromatographic method development in an analytical quality by design framework. Journal of Liquid Chromatography \& Related Technologies 2013;36(18):2612-38. DOI: 10.1080/10826076.2013.765457.

5. Karmarkar S, Garber R, Genchanok Y, George S, Yang X, Hammond R. Quality by design (QbD) based development of a stability indicating HPLC method for drug and impurities. J Chromatogr Sci. 2011;49(6):439-46.
6. Gheshlaghi R, Scharer JM, Moo-Young M, Douglas PL. Application of statistical design for the optimization of amino acid separation by reverse-phase HPLC. Anal Biochem. 2008;383(1):93-102. Doi: 10.1016/j.ab.2008.07.032.

7. Myers RH, Montgomery DC. Response Surface Methodology. Bloomfield P, Cressie NAC, Fisher NI, Johnstone IM, Kadane JB, Ryan LM, Scott DW, Silverman BW, Smith AFM, Teugels JL, editors: John Wiley \& Sons, INC.; 2002.

8. Zhou Y, Song JZ, Choi FF, Wu HF, Qiao CF, Ding LS, et al. An experimental design approach using response surface techniques to obtain optimal liquid chromatography and mass spectrometry conditions to determine the alkaloids in Meconopsi species. J Chromatogr A. 2009;1216(42):7013-23. Doi: 10.1016/j.chroma.2009.08.058.

9. Marengo E, Gianotti V, Angioi S, Gennaro MC. Optimization by experimental design and artificial neural networks of the ioninteraction reversed-phase liquid chromatographic separation of twenty cosmetic preservatives. J Chromatogr A. 2004;1029(1-2):5765.

10. Debrus B, Lebrun P, Kindenge JM, Lecomte F, Ceccato A, Caliaro $\mathrm{G}$, et al. Innovative high-performance liquid chromatography method development for the screening of 19 ant malarial drugs based on a generic approach, using design of experiments, independent component analysis and design space. J Chromatogr A. 2011;1218(31):5205-15. Doi: 10.1016/j.chroma.2011.05.102.

11. Chloupek RC, Hancock WS, Snyder LR. Computer simulation as a tool for the rapid optimization of the high-performance liquid chromatographic separation of a tryptic digest of human growth hormone. J Chromatogr. 1992;594(1-2):65-73.

12. Dolan JW, Lommen DC, Snyder LR. Dry Lab computer simulation for high-performance liquid chromatographic method development. II. Gradient elution. J Chromatogr. 1989;485:91-112.

13. Dolan JW, Snyder LR, Djordjevic NM, Hill DW, Saunders DL, Van Heukelem L. et al. Simultaneous variation of temperature and gradient steepness for reversed-phase high-performance liquid chromatography method development. I. Application to 14 different samples using computer simulation. J Chromatogr A. 1998;803(12):1-31.

14. Dolan JW, Snyder LR, Wolcott RG, Haber P, Baczek T, Kaliszan R, et al. Reversed-phase liquid chromatographic separation of complex samples by optimizing temperature and gradient time III. Improving the accuracy of computer simulation. J Chromatogr A. 1999;857(12):41-68.

15. Fekete S, Fekete J, Molnár I, Ganzler K. Rapid high performance liquid chromatography method development with high prediction accuracy, using $5 \mathrm{~cm}$ long narrow bore columns packed with sub-2microm particles and Design Space computer modeling. J Chromatogr A. 2009;1216(45):7816-23. Doi: 10.1016/j.chroma.2009.09.043.

16. Jayaraman K, Alexander AJ, Hu Y, Tomasella FP. A stepwise strategy employing automated screening and Dry Lab modeling for the development of robust methods for challenging high performance liquid chromatography separations: a case study. Anal Chim Acta. 2011;696(1-2):116-24. Doi: 10.1016/j.aca.2011.04.010.

17. Krisko RM, McLaughlin K, Koenigbauer MJ, Lunte CE. Application of a column selection system and DryLab software for high-performance liquid chromatography method development. J Chromatogr A. 2006;1122(1-2):186-93.

18. Lämmerhofer M, Di Eugenio P, Molnar I, Lindner W. Computerized optimization of the high-performance liquid chromatographic 
enantioseparation of a mixture of 4-dinitrophenyl amino acids on a quinine carbamate-type chiral stationary phase using DRYLAB. J Chromatogr B Biomed Sci Appl. 1997;689(1):123-35.

19. Lewis JA, Lommen DC, Raddatz WD, Dolan JW, Snyder LR, Molnar I Computer simulation for the prediction of separation as a function of $\mathrm{pH}$ for reversed-phase high-performance liquid chromatography. I. Accuracy of a theory-based model. J Chromatogr. 1992;592(12):183-95

20. Li W, Rasmussen HT. Strategy for developing and optimizing liquid chromatography methods in pharmaceutical development using computer-assisted screening and Plackett-Burman experimental design. J Chromatogr A. 2003 ;1016(2):165-80.

21. Molnar I. Computerized design of separation strategies by reversedphase liquid chromatography: development of Dry Lab software. J Chromatogr A. 2002;965(1-2):175-94.

22. Schellinger AP, Mao Y, Carr PW. Use of DRYLAB to compare octadecylsilane and carbon supports for reversed-phase chromatography of triazine herbicide test solutes. Anal Bioanal Chem. 2002;373(7):587-94.

23. Schmidt AH, Molnar I. Computer-assisted optimization in the development of a high-performance liquid chromatographic method for the analysis of kava pyrones in Piper methysticum preparations. J Chromatogr A. 2002 ;948(1-2):51-63.

24. Wang X, Stoll DR, Schellinger AP, Carr PW. Peak capacity optimization of peptide separations in reversed-phase gradient elution chromatography: fixed column format. Anal Chem. 2006;78(10):3406-16.

25. Snyder LR, Kirkland JJ, Dolan JW. Introduction to modern liquid chromatography. 3rd ed: John Wiley \& Sons, Inc, 2009.

26. Carr PW, Stoll DR, Wang X. Perspectives on recent advances in the speed of high-performance liquid chromatography. Anal Chem. 2011;83(6):1890-900. Doi: 10.1021/ac102570t.

27. Carr PW, Wang X, Stoll DR. Effect of pressure, particle size, and time on optimizing performance in liquid chromatography. Anal Chem. 2009;81(13):5342-53. Doi: 10.1021/ac9001244.

28. Snyder LR, Dolan JW. High-Performance Gradient Elution: The Practical Application of the Linear-Solvent-Strength Model. Hoboken: Wiley \& Sons; 2007.

29. Gu H, Huang Y, Carr PW. Peak capacity optimization in comprehensive two dimensional liquid chromatography: a practical approach. J Chromatogr A. 2011;1218(1):64-73. Doi: 10.1016/j. chroma.2010.10.096.

30. Quality by Deisn (QbS) Using JMP Software Course Notes: SAS Institute; 2014.

31. Horvath C, Lin H. Movement and Band Spreading of Unsorbed Species in Liquid Chromatography. Journal of Chromatography1976;126:401-20.

32. Csaba Horva'th, Hung-Jye Lin. Band Spreading in Liquid Chromatography- General Plate Height Equation and a Method for the Evaluation of the Individual Plate Height Contributions. Journal of Chromatography1978;149:43-70. Doi:10.1016/S00219673(00)80978-4.

33. Thompson JD, Brown JS, Carr PW. Dependence of thermal mismatch broadening on column diameter in high-speed liquid chromatography at elevated temperatures. Anal Chem. 2001;73(14):3340-7.

34. Zhang Y, Wang X, Mukherjee P, Petersson P. Critical comparison of performances of superficially porous particles and sub-2 microm particles under optimized ultra-high pressure conditions. J Chromatogr A. 2009 ;1216(21):4597-605. Doi: 10.1016/j. chroma.2009.03.071.

35. Thompson JD, Carr PW. High-speed liquid chromatography by simultaneous optimization of temperature and eluent composition. Anal Chem. 2002;74(16):4150-9.

36. Boswell PG, Stoll DR, Carr PW, Nagel ML, Vitha MF, Mabbott GA. An Advanced, Interactive, High-Performance Liquid Chromatography Simulator and Instructor Resources. J Chem Educ. 2013;90(2):198202.

37. Li J, Carr PW. Accuracy of Empirical Correlations for Estimating Diffusion Coefficients in Aqueous Organic Mixtures. Anal Chem. 1997;69(13):2530-6.

38. Li J, Carr PW. Estimating Diffusion Coefficients for Alkylbenzenes and Akylphenones in Aque-ous Mixtures with Acetonitrile and Methanol. Anal Chem. 1997;69(13):2550-3.

39. Cabooter D, Billen J, Terryn H, Lynen F, Sandra P, Desmet G. Detailed characterisation of the flow resistance of commercial sub-2 micrometer reversed-phase columns. J Chromatogr A. 2008;1178(12):108-17.

40. Cabooter D, Fanigliulo A, Bellazzi G, Allieri B, Rottigni A, Desmet G. Relationship between the particle size distribution of commercial fully porous and superficially porous high-performance liquid chromatography column packings and their chromatographic performance. J Chromatogr A. 2010;1217(45):7074-81. Doi: 10.1016/j.chroma.2010.09.008.

41. Gritti F, Guiochon G. Comparative study of the performance of columns packed with several new fine silica particles. Would the external roughness of the particles affect column properties? J Chromatogr A. 2007;1166(1-2):30-46.

42. Cabooter D, Decrop W, Eeltink S, Swart R, Ursem M, Lestremau F, et al. Automatic column coupling system to operate chromatographic supports closer to their kinetic performance limit and to enhance method development. Anal Chem. 2010;82(3):1054-65. Doi: 10.1021/ac902404v.

43. Cabooter D, Billen J, Terryn H, Lynen F, Sandra P, Desmet G. Kinetic plot and particle size distribution analysis to discuss the performance limits of sub-2 microm and supra-2 microm particle columns. J Chromatogr A. 2008;1204(1):1-10. Doi: 10.1016/j. chroma.2008.07.007.

44. Dai J, Carr PW. Role of ion pairing in anionic additive effects on the separation of cationic drugs in reversed-phase liquid chromatography. J Chromatogr A. 2005;1072(2):169-84.

45. Dai J, Carr PW. Effect of mobile phase anionic additives on selectivity, efficiency, and sample loading capacity of cationic drugs in reversedphase liquid chromatography. J Chromatogr A. 2009;1216(39):6695705. Doi: 10.1016/j.chroma.2009.07.062.

46. Dai J, Mendonsa SD, Bowser MT, Lucy CA, Carr PW. Effect of anionic additive type on ion pair formation constants of basic pharmaceuticals. J Chromatogr A. 2005;1069(2):225-34.

47. Samuelsson J, Franz A, Stanley BJ, Fornstedt T. Thermodynamic characterization of separations on alkaline-stable silica-based C18 columns: why basic solutes may have better capacity and peak performance at higher pH. J Chromatogr A. 2007;1163(1-2):177-89.

48. Snyder LR, Dolan JW, Carr PW. The hydrophobic-subtraction model of reversed-phase column selectivity. J Chromatogr A. 2004;1060(1- 
2):77-116.

49. Stoll DR, Wang X, Carr PW. Comparison of the practical resolving power of one- and two-dimensional high-performance liquid chromatography analysis of metabolomic samples. Anal Chem. 2008;80(1):268-78.

50. Zhu PL, Snyder LR, Dolan JW, N.M.Djordjevic, Hill DW, L.C.Sander, et al. Predicting Separation as a Function of Temperature and Gradient Condition. Combined use of Temperature and Solvent Strength in Reversed-Phase Gradient Elution. Journal of Chromatography1996;756:21-39.
51. LoBrutto R, Jones A, Kazakevich YV, McNair HM. Effect of the eluent $\mathrm{pH}$ and acidic modifiers in high-performance liquid chromatography retention of basic analytes. J Chromatogr A. 2001;913(1-2):173-87.

52. LoBrutto R, Jones A, Kazakevich YV. Effect of counter-anion concentration on retention in high-performance liquid chromatography of protonated basic analytes. J Chromatogr A. 2001;913(1-2):189-96.

53. Cecchi T, Passamonti P. Retention mechanism for ion-pair chromatography with chaotropic reagents. J Chromatogr A. 2009;1216(10):1789-97. Doi: 10.1016/j.chroma.2008.10.031. 